OPEN ACCESS

Edited by:

John D. Imig,

Medical College of Wisconsin

United States

Reviewed by:

Hui-Ching Lin,

National Yang-Ming University, Taiwan

Artiom Gruzdev,

National Institute of Environmental Health Sciences (NIEHS),

United States

Aldrin V. Gomes,

University of California, Davis,

United States

${ }^{*}$ Correspondence:

Kenji Hashimoto

hashimoto@faculty.chiba-u.jp

Specialty section:

This article was submitted to Translational Pharmacology,

a section of the journal

Frontiers in Pharmacology

Received: 08 November 2018

Accepted: 14 January 2019

Published: 30 January 2019

Citation:

Hashimoto K (2019) Role of Soluble Epoxide Hydrolase in Metabolism of PUFAs in Psychiatric

and Neurological Disorders.

Front. Pharmacol. 10:36

doi: 10.3389/fphar.2019.00036

\section{Role of Soluble Epoxide Hydrolase in Metabolism of PUFAs in Psychiatric and Neurological Disorders}

\author{
Kenji Hashimoto* \\ Division of Clinical Neuroscience, Center for Forensic Mental Health, Chiba University, Chiba, Japan
}

Inflammation plays a key role in the pathogenesis of a number of psychiatric and neurological disorders. Soluble epoxide hydrolases (sEH), enzymes present in all living organisms, metabolize epoxy fatty acids (EpFAs) to corresponding 1,2-diols by the addition of a molecule of water. Accumulating evidence suggests that $\mathrm{sEH}$ in the metabolism of polyunsaturated fatty acids (PUFAs) plays a key role in inflammation. Preclinical studies demonstrated that protein expression of $\mathrm{sEH}$ in the prefrontal cortex, striatum, and hippocampus from mice with depression-like phenotype was higher than control mice. Furthermore, protein expression of sEH in the parietal cortex from patients with major depressive disorder was higher than controls. Interestingly, Ephx2 knockout $(\mathrm{KO})$ mice exhibit stress resilience after chronic social defeat stress. Furthermore, the $\mathrm{sEH}$ inhibitors have antidepressant effects in animal models of depression. In addition, pharmacological inhibition or gene $\mathrm{KO}$ of sEH protected against dopaminergic neurotoxicity in the striatum after repeated administration of MPTP (1-methyl-4-phenyl1,2,3,6-tetrahydropyridine) in an animal model of Parkinson's disease (PD). Protein expression of $\mathrm{sEH}$ in the striatum from MPTP-treated mice was higher than control mice. A number of studies using postmortem brain samples showed that the deposition of protein aggregates of $\alpha$-synuclein, termed Lewy bodies, is evident in multiple brain regions of patients from PD and dementia with Lewy bodies (DLB). Moreover, the expression of the sEH protein in the striatum from patients with DLB was significantly higher compared with controls. Interestingly, there was a positive correlation between $\mathrm{sEH}$ expression and the ratio of phosphorylated $\alpha$-synuclein to $\alpha$-synuclein in the striatum. In the review, the author discusses the role of sEH in the metabolism of PUFAs in inflammation-related psychiatric and neurological disorders.

\footnotetext{
Keywords: $\alpha$-synuclein, cytochrome P450, dementia of Lewy bodies, depression, epoxy fatty acids, inflammation, Parkinson's disease, stress resilience
}

\section{INTRODUCTION}

Polyunsaturated fatty acids (PUFAs) are generally considered to be necessary for maintaining normal physiology (Jump, 2002; Bazinet and Layé, 2014; Layé et al., 2018). PUFAs are known to regulate both the structure and the function of neurons, glial cells, and endothelial cells in the brain (Bazinet and Layé, 2014; Layé et al., 2018). Importantly, PUFAs 
need to be provided by the diet since they cannot be produced in mammals. There are two main families ( $\omega-3$ and $\omega-6)$ of PUFAs. Linoleic acid, the predominant plant-derived dietary $\omega$ 6 PUFA, is a precursor of arachidonic acid (ARA). $\alpha$-linolenic acid, the predominant plant-derived dietary $\omega-3$ PUFA, is a precursor of eicosapentaenoic acid (EPA) and docosahexaenoic acid (DHA).

Polyunsaturated fatty acids (PUFAs) are metabolized into bioactive derivatives by the main enzymes such as cyclooxygenases (COXs), lipoxygenases (LOXs), and cytochrome P450s (CYPs) (Imig and Hammock, 2009; Arnold et al., 2010; Imig, 2012, 2018; Morisseau and Hammock, 2013; Bazinet and Layé, 2014; Urquhart et al., 2015; Westphal et al., 2015; Figure 1). The COX pathway leads to the formation of prostaglandins, prostacyclines and thromboxanes, the LOX pathway leads to leukotrienes, lipoxins, and hydroxyleicosatetraenoic acids (HETEs). The CYP pathway leads to 20-HETE by CYP hydroxylases, and epoxy fatty acids (EpFAs) such as epoxy-eicosatrienoic acids (EETs) and epoxydocosapentaenoic acids (EDPs) by CYP epoxygenases (Figure 1).

In the review, the author would like to discuss the role of soluble epoxide hydrolase $(\mathrm{sEH})$ in the CYP-mediated metabolism of PUFAs which might be involved in the pathogenesis of psychiatric and neurological disorders. Furthermore, we also refer to the clinical significance of $\mathrm{sEH}$ inhibitors for these disorders.



FIGURE 1 | Overview of metabolism of polyunsaturated fatty acids (PUFAs). PUFAs such as arachidonic acid (ARA), eicosapentaenoic acid (EPA), and docosahexaenoic acid (DHA), are converted to prostaglandins, prostacyclins, and thromboxanes by cyclooxygenase (COX). PUFAs are also converted to leukotrienes, lipoxins, hydroxyeicosatetraenoic acids (HETEs) by lipoxygenase (LOX). Moreover, PUFAs are converted to hydroxyeicosatetraenoic acids (HETEs), including 20-hydroxyeicosatetraenoic acid (20-HETE), and epoxy fatty acids (EpFAs), including epoxyeicosatrienoic acids (EETs) and epoxydocosapentaenoic acids (EDPs), by cytochrome P450 (CYP) hydroxylases and CYP epoxygenases, respectively. EpFAs (e.g., EETs, EDPs) are converted to their corresponding 1,2-diols (e.g., dihydroxyeicosatrienoic acids (DHETs), dihydroxydocosapentaenoic acids [DiHDPAs]) by soluble epoxide hydrolase (sEH). (modified from Morisseau and Hammock, 2013 and Hashimoto, 2016).

\section{SOLUBLE EPOXIDE HYDROLASE IN CYP SYSTEM}

The CYP system is a superfamily of enzymes, which are involved in the metabolism of exogenous and endogenous compounds. The CYP in the eicosanoid pathway was first described in 1980 and is comprised of two enzymatic pathways such as hydroxylases and epoxygenases. The CYP isoforms metabolize a number of $\omega-3$ and $\omega-6$ PUFAs, including ARA, EPA and DHA into bioactive lipid mediators, termed eicosanoids (Imig and Hammock, 2009; Imig, 2012, 2018; Morisseau and Hammock, 2013; Urquhart et al., 2015; Westphal et al., 2015; Jamieson et al., 2017). The CYP system produces both the pro-inflammatory, terminally hydroxylated metabolite 20-HETE and the antiinflammatory EpFAs, including EETs from ARA and EDPs from DHA (Figure 1).

In contrast, EpFAs such as EETs, and EDPs are rapidly metabolized by a number of pathways including the soluble epoxide hydrolase (sEH) (Imig and Hammock, 2009; Morisseau and Hammock, 2013). The sEH was first identified in the cytosolic fraction of mouse liver through its activity on epoxide containing substances such as juvenile hormone and lipid epoxides (Hammock et al., 1976; Gill and Hammock, 1980; Ota and Hammock, 1980). Human sEH is a $62 \mathrm{kDa}$ enzyme composed of two domains separately by a short proline-rich linker (Harris and Hammock, 2013). The N-terminal domain has a phosphatase activity that hydrolyzes lipid phosphates, while the C-terminal domain has an epoxide hydrolase activity that converts epoxides to their corresponding diols (Newman et al., 2003). The human EPHX2 gene codes for the sEH protein is widely expressed in a number of tissues, including the liver, lungs, kidney, heart, brain, adrenals, spleen, intestines, urinary bladder, placenta, skin, mammary gland, testis, leukocytes, vascular endothelium, and smooth muscle. Interestingly, the sEH protein is most highly expressed in the liver and kidney (Gill and Hammock, 1980; Newman et al., 2005; Imig, 2012).

Accumulating evidence suggests that EETs, EDPs and some other EpFAs have potent anti-inflammatory properties (Wagner et al., 2014, 2017; López-Vicario et al., 2015) which are implicated in the pathogenesis of a number of psychiatric and neurological disorders (Denis et al., 2015; Hashimoto, 2015, 2016, 2018; Gumusoglu and Stevens, 2018; Polokowski et al., 2018).

\section{INFLAMMATION IN DEPRESSION AND SEH}

Depression, one of the most common disorders in the world, is a major psychiatric disorder with a high rate of relapse. The World Health Organization (WHO) estimates that more than 320 million individuals of all ages suffer from depression (World Health Organization [WHO], 2017). Multiple lines of evidence demonstrate inflammatory processes in the pathophysiology of depression and in the antidepressant actions of the certain compounds (Dantzer et al., 2008; Miller et al., 2009, 2017; Raison et al., 2010; Hashimoto, 2015, 2016, 2018; Mechawar and Savitz, 2016; Miller and Raison, 2016; Zhang et al., 
2016a,b, 2017b,a). Meta-analysis showed higher levels of proinflammatory cytokines in the blood of drug-free or medicated depressed patients compared to healthy controls (Dowlati et al., 2010; Young et al., 2014; Haapakoski et al., 2015; Eyre et al., 2016; Köhler et al., 2018). Collectively, it is likely that inflammation plays a key role in the pathophysiology of depression.

Several reports using meta-analysis demonstrated that $\omega-3$ PUFAs could reduce depressive symptoms beyond placebo (Lin et al., 2010, 2017; Sublette et al., 2011; Mello et al., 2014; Grosso et al., 2016; Hallahan et al., 2016; Mocking et al., 2016; Sarris et al., 2016; Bai et al., 2018; Hsu et al., 2018). Dietary intake of $\omega-3$ PUFAs is known to be associated with lower risk of depression. Importantly, EPA-rich $\omega-3$ PUFAs could be recommended for the treatment of depression (Sublette et al., 2011; Mocking et al., 2016; Sarris et al., 2016). Importantly, brain EPA levels are 250300-fold lower than DHA compared to about 4- (plasma), 5(erythrocyte), 14- (liver), and 86-fold (heart) lower levels of EPA versus DHA (Chen and Bazinet, 2015; Dyall, 2015).

Given the role of inflammation in depression, it is likely that $s E H$ might contribute to the pathophysiology of depression. A single injection of lipopolysaccharide (LPS) is known to produce depression-like phenotypes in rodents after sickness behaviors (Dantzer et al., 2008; Zhang et al., 2014, 2016a, 2017b; Ma et al., 2017; Yang et al., 2017). Ren et al. (2016) reported that the sEH inhibitor TPPU [1-(1propionylpiperidin-4-yl)-3-(4-(trifluoromethoxy)phenyl)urea]

(Figure 2) conferred prophylactic and antidepressant effects in the LPS-induced inflammation model of depression while the current antidepressants showed no therapeutic effects in this model (Zhang et al., 2014). Chronic social defeat stress (CSDS) model of depression is widely used as an animal model of depression (Nestler and Hyman, 2010; Golden et al., 2011; Yang et al., 2015, 2017, 2018). Pretreatment with TPPU before social defeat stress showed resilience to CSDS. In addition, TPPU showed rapid antidepressant effects in susceptible mice after CSDS (Ren et al., 2016). Interestingly, the sEH KO mice showed stress resilience to repeated social defeat stress. Increased brain-derived neurotrophic factor (BDNF) and its receptor TrkB signaling in the prefrontal cortex and hippocampus of the $\mathrm{KO}$ mice might be responsible for stress resilience (Ren et al., 2016). Furthermore, repeated treatment with TPPU for 7 days increased interaction time of socially defeated mice in a CSDS model, and improvement by TPPU was blocked by TrkB antagonist K252a (Wu et al., 2017), suggesting a role of BDNF-TrkB signaling in TPPU's beneficial effects. Interestingly, higher protein levels of $s E H$ were shown in the brain regions of mice with a depression-like phenotype in the inflammation and CSDS models, suggesting that increased levels of sEH may play a role in depression-like phenotypes in rodents (Ren et al., 2016). We found higher sEH protein levels in the parietal cortex (Brodmann area 7) from patients with major depressive disorder, pointing to a possible role for increased $\mathrm{sEH}$ levels in depression (Ren et al., 2016). Taken together, this study highlights a key function for $\mathrm{sEH}$ in the pathophysiology of depression, and for its inhibitors as potential therapeutic or prophylactic drugs for depression (Hashimoto, 2016; Ren et al., 2016; Swardfager et al., 2018; Figure 3).

A study using euthymic patients with a history of major depressive disorder with seasonal depression showed changes in CYP- and sEH-derived eicosanoids in patients with winter depression (Hennebelle et al., 2017). The $\omega-6$ derived sEH product 12,13-DiHOME [12,13-dihydroxy-9-octadecenoic acid] increased in winter depression. Total 14,15-EpETE [14,15epoxy-5Z,8Z,11Z,17Z-eicosatetraenoic acid], a sEH substrate, as well as sEH-derived free 14,15-DiHETrE [14,15-dihydroxy5Z,8Z,11Z- eicosatrienoic acid], decreased during winter<smiles>CCC(=O)N1CCC(NC(=O)Nc2ccc(OC(F)(F)F)cc2)CC1</smiles>

TPPU<smiles>COc1ccc(CNC(=O)NCc2ccc(OC)cc2)cc1</smiles>

MMU<smiles>O=C(O)CCCCCCCCCCCCC(=O)NC12CC3CC(CC(C3)C1)C2</smiles>

AUDA<smiles>C=CCc1ccc(O)c(-c2ccc(O)c(CC=C)c2)c1</smiles>

Honokiol

FIGURE 2 | Chemical structure of SEH inhibitors TPPU, AUDA, MMU, and honokiol. 


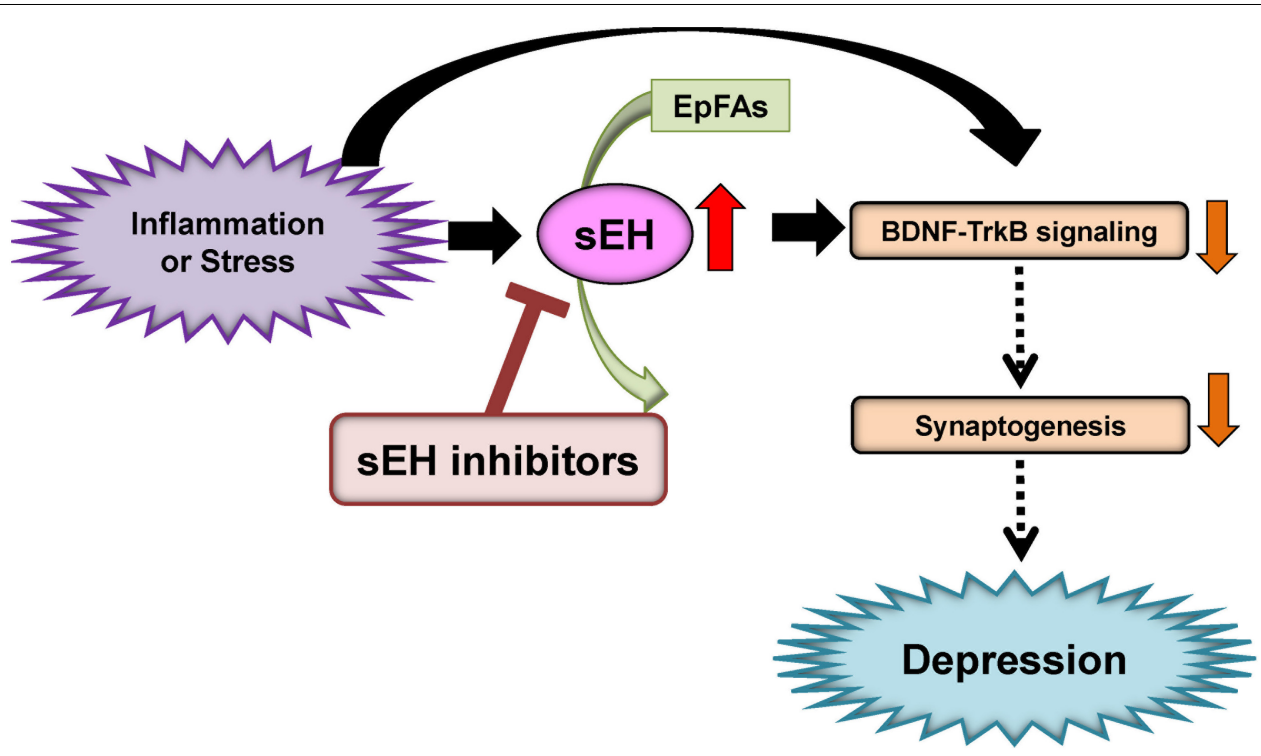

FIGURE 3 | Proposed mechanism of the role of sEH in depression. Inflammation or stress can increase the expression of sEH in the brain, resulting in enhanced metabolism of anti-inflammatory PUFA epoxides (EpFAs). Subsequently, increased expression of sEH can decrease BDNF-TrkB signaling and synaptogenesis, leading to depressive symptoms. The sEH inhibitors may have antidepressant actions in depressed patients. (modified from Hashimoto, 2016).

compared to summer-fall, while sEH-derived total 7,8-DiHDPE [7,8-dihydroxy-4Z,10Z,13Z,16Z,19Z-docosapentaenoic acid], total 19,20-DiHDPE $\quad[19,20$-dihydroxy-4Z,7Z,10Z,13Z,16Zdocosapentaenoic acid], and total 12,13-DiHOME [12,13-dihydroxy-9Z-octadecenoic acid] were increased during winter. These findings suggest that seasonal shifts in $\omega-6$ and $\omega-3$ PUFAs metabolism mediated by sEH may underlie inflammatory states in symptomatic depression with seasonal pattern (Hennebelle et al., 2017). Given the crucial role of $s E H$ in the metabolism of $\omega-3$ PUFAs, $\omega-3$ PUFAs such as EPA in combination with a $\mathrm{sEH}$ inhibitor would be a novel therapeutic approach for depression (Figure 3).

\section{EATING DISORDERS AND ADHD}

Anorexia nervosa (AN) is a serious eating disorder characterized by the persistent restriction of energy intake, intense fear of gating weight, and distribution in self-perceived weight or shape. The Epoxide Hydrolase 2 (EPHX2) gene was found to harbor several common and rare risk variants for $\mathrm{AN}$ (Scott-Van Zeeland et al., 2014). Subsequently, the patients with AN show elevated plasma levels of $\omega-3$ PUFAs (ARA, EPA, DHA) compared to controls (Shih et al., 2016). Interestingly, 15,16-DiHODE [15,16-dihydroxy-9Z,12Z-octadecadienoic acid]/15,16-EpODE [15,16-epoxy-9Z,12Z-octadecadienoic acid] ratio derived from ARA and 19,20-DiHDPE [19,20-dihydroxy4Z,7Z,10Z,13Z,16Z- docosapentaenoic acid]/19,20-EpDPE [19,20-epoxy-4Z,7Z,10Z,13Z,16Z-docosapentaenoic acid] ratio derived from DHA in AN patients were higher than controls, suggesting a higher in vivo sEH activity, concentration, or efficiency in AN (Shih et al., 2016; Shih, 2017). These data suggest the role of EPHX2-associated eicosanoid dysregulations in AN.
Collectively, sEH inhibitors might be potential therapeutic drugs for AN (Shih et al., 2016; Shih, 2017).

Attention deficit hyperactivity disorder (ADHD) is one of the most common psychiatric disorders affecting children. Symptoms of ADHD include inattention, hyperactivity and impulsivity. However, biological mechanisms underlying ADHD remain unknown. A meta-analysis shows that children and youth with ADHD have elevated ratios of both blood $\omega-6 / \omega-3$ PUFAs compared to controls (LaChance et al., 2016), suggesting an elevated $\omega-6 / \omega-3$, and more specifically ARA/EPA ratio may represent the underlying disturbance in essential PUFAs levels in patients with ADHD. A recent meta-analysis shows that children and adolescents with ADHD have lower levels of DHA, EPA, and total $\omega-3$ PUFAs (Chang et al., 2018). Furthermore, supplementation of $\omega-3$ PUFAs, particularly with high doses of EPA, was modestly effective in the treatment of ADHD (Bloch and Qawasmi, 2011; Chang et al., 2018). Collectively, it is of great interest to study whether blood levels of EpFAs and their corresponding diols are altered in the patients with ADHD. Furthermore, it is also interesting to investigate the role of $\mathrm{sEH}$ in the pathogenesis of ADHD since there are no reports showing the role of $\mathrm{sEH}$ in ADHD.

\section{INFLAMMATION IN PARKINSON'S DISEASE AND SEH}

Parkinson's disease (PD) is the second most common neurodegenerative disease after Alzheimer's disease. Although the precise pathogenesis of $\mathrm{PD}$ is unknown, the pathological hallmark of PD involves the progressive loss of dopaminergic neurons in the substantia nigra (SN) (Kalia and Lang, 2015; 
Ascherio and Schwarzschild, 2016). In addition, the deposition of aggregates of $\alpha$-synuclein, termed Lewy bodies, is evident in multiple brain regions of patients from PD and dementia with Lewy bodies (DLB) (Spillantini et al., 1997). There are, to date, no agents with a disease-modifying or neuroprotective indication for PD has been approved (Dehay et al., 2015). Interestingly, it is noteworthy that PD or DLB patients have depressive symptoms (Cummings, 1992; Takahashi et al., 2009; Goodarzi et al., 2016; Schapira et al., 2017), indicating that management of depression in these patients is also important. Therefore, the development of new drugs possessing disease-modifying and/or neuroprotective properties is unmet medical need.

$\omega-3$ PUFAs appear to be neuroprotective for several neurological disorders. It is reported that dietary intake of PUFAs is associated with lower risk of PD (Kamel et al., 2014; Seidl et al., 2014). MPTP (1-methyl-4-phenyl-1,2,3,6-tetrahydropyridine)induced neurotoxicity in the striatum and $\mathrm{SN}$ has been widely used as an animal model of PD (Sedelis et al., 2001; JacksonLewis and Przedborski, 2007). A diet rich in EPA diminished MPTP-induced hypokinesia in mice and ameliorated procedural memory deficit (Luchtman et al., 2012). Recently, we reported that MPTP-induced neurotoxicity [e.g., loss of dopamine transporter (DAT), loss of tyrosine hydrolase (TH)-positive cells, increased endoplasmic reticulum (ER) stress] in the striatum and $\mathrm{SN}$ was attenuated after subsequent repeated oral administration of TPPU (Ren et al., 2018). MPTP-induced loss of THpositive cells in the $\mathrm{SN}$ is also attenuated by pretreatment with another sEH inhibitor, AUDA [12-((tricyclo(3.3.1.13,7)dec-1ylamino)carbonyl)amino)-dodecanoic acid] (Figure 2), although posttreatment with AUDA did not attenuate MPTP-induced neurotoxicity (Qin et al., 2015). Furthermore, deletion of the sEH gene protected against MPTP-induced neurotoxicity in the mouse striatum (Huang et al., 2018; Ren et al., 2018), while overexpression of $\mathrm{sEH}$ in the striatum significantly enhanced

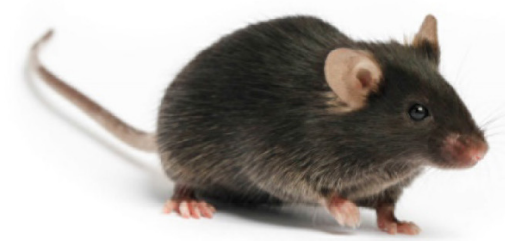

MPTP Injections

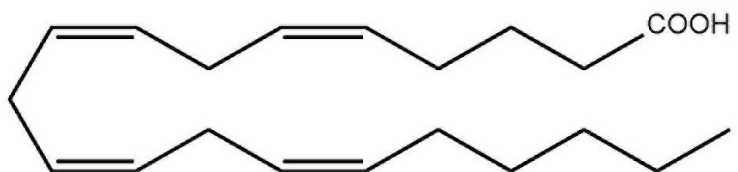

ARA

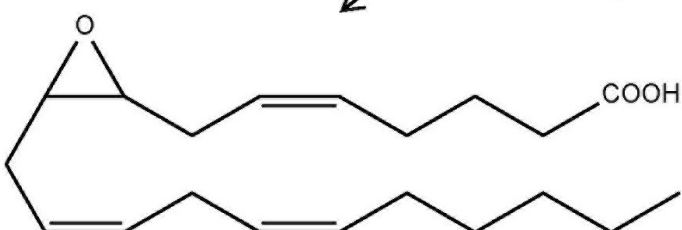

sEH

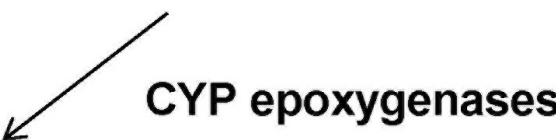

8,9-EpETrE

\section{8,9-DiHETrE}

\section{Neurotoxicity in the striatum and SN}

FIGURE 4 | Possible mechanism of role of sEH in the MPTP-induced neurotoxicity. 8,9-EpETrE is prepared from ARA by CYP epoxygenases, and it is metabolized by sEH into 8,9-DiHETrE. Repeated MPTP injections into mice caused increased sEH expression in the striatum, resulting the reduction of anti-inflammatory 8,9-EpETrE in the striatum. Finally, these events cause dopaminergic neurotoxicity in the striatum and SN. Pharmacological inhibition or knock-out of sEH could protect against MPTP-induced neurotoxicity in the striatum and SN. 
MPTP-induced neurotoxicity (Ren et al., 2018). Moreover, the expression of the $\mathrm{sEH}$ protein in the striatum from MPTP-treated mice was significantly higher than control group. Interestingly, there was a positive correlation between sEH expression and phosphorylation of $\alpha$-synuclein in the striatum, suggesting that sEH may play a role in the phosphorylation of $\alpha$-synuclein in the mouse striatum (Ren et al., 2018). Oxylipin analysis showed reduced levels of 8,9-epoxy-5Z,11Z,14Z-eicosatrienoic acid (8,9-EpETrE) prepared from ARA in the striatum of MPTPtreated mice, suggesting increased activity of $\mathrm{sEH}$ in this region (Figure 4).

Deposition of $\alpha$-synuclein has been shown in multiple brain regions of PD and DLB patients (Spillantini et al., 1997). Interestingly, the high levels of DHA in brain areas containing $\alpha$-synuclein in PD patients may support the possible interaction between $\alpha$-synuclein and DHA (Fecchio et al., 2018). Protein levels of $\mathrm{sEH}$ in the striatum from DLB patients were significantly higher than those of the controls, whereas protein levels of DAT and $\mathrm{TH}$ in the striatum from DLB patients were significantly lower than those of controls (Ren et al., 2018). Furthermore, the ratio of phosphorylated $\alpha$-synuclein to $\alpha$-synuclein in the striatum from DLB patients was significantly higher than that of controls (Ren et al., 2018). Interestingly, there was a positive correlation between $\mathrm{sEH}$ levels and the ratio of phosphorylated $\alpha$-synuclein to $\alpha$-synuclein in all subjects (Ren et al., 2018). Collectively, it is likely that increased $\mathrm{sEH}$ and resulting increase in phosphorylation of $\alpha$-synuclein may play a role in the pathogenesis of PD.
The PARK2 is one of the familial forms of PDs caused by a mutation in the PARKIN gene (Imaizumi et al., 2012). In addition, the expression of EPHX2 mRNA in human PARK2 iPSC-derived neurons was higher than that of healthy control group. Treatment with TPPU protected against apoptosis in human PARK2 iPSC-derived neurons (Ren et al., 2018). These findings suggest that increased activity of $\mathrm{sEH}$ in the striatum plays a key role in the pathogenesis of neurological disorders such as PD and DLB although common polymorphisms within EPHX2 do not appear to be important risk factors for PD (Farin et al., 2001). Accumulation of aggregated $\alpha$-synuclein is the pathological hallmark of PD and DLB although its precise role is not understood. Our data suggest a possible interaction between phosphorylation of $\alpha$-synuclein and $\mathrm{sEH}$ expression in the striatum from DLB patients. Taken all together, it is likely that $s E H$ could represent a promising therapeutic target for $\alpha$-synuclein-related neurological disorders such as PD and DLB (Borlongan, 2018; Ren et al., 2018; Figure 5). In addition, there are also several approaches (e.g., a smallinterfering RNA, immunotherapies, enhancement of autophagy) to reduce $\alpha$-synuclein production (Stoker et al., 2018).

\section{CONCLUSION REMARKS AND FUTURE PERSPECTIVE}

Many patients with depression become chronically ill, with several relapses or later recurrences, following initial short-term improvement or remission. Relapses occur at a rate of over 85

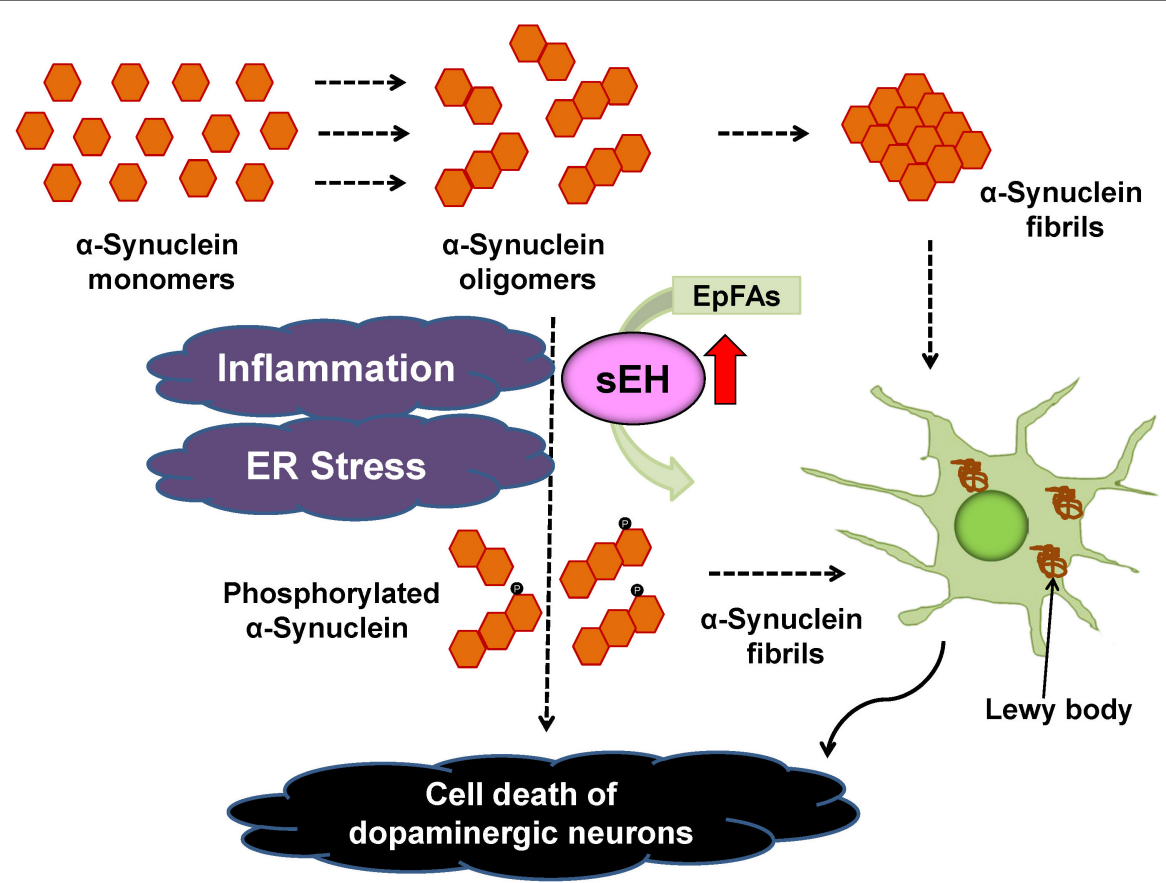

FIGURE 5 | Proposed mechanism of the role of sEH in the pathogenesis of PD and DLB. Inflammation and ER stress can increase the expression of sEH in the striatum, resulting in enhanced metabolism of anti-inflammatory EpFAs, leading to increased phosphorylation of $\alpha$-synuclein (Ren et al., 2018). The sEH inhibitors may prevent the progression of aggregation of phosphorylated $\alpha$-synuclein in the brain. 
percent within a decade of an index depressive episode (Forte et al., 2015; Sim et al., 2015). Therefore, the prevention of relapse and recurrence is important in the management of depression. Taken together, it seems that sEH inhibitors could be prophylactic drugs to prevent or minimize relapses triggered by inflammation and/or stress in remitted patients with depression (Hashimoto, 2016; Ren et al., 2016). In addition, given the comorbidity of depressive symptoms in PD or DLB patients (Cummings, 1992; Takahashi et al., 2009; Goodarzi et al., 2016; Schapira et al., 2017), it is also likely that sEH inhibitors may serve as prophylactic drugs to prevent the progression of PD or DLB in patients.

Some natural compounds with sEH inhibitory action were reported. MMU [1,3-bis (4-methoxybenzyl)urea](Figure 2), the most abundant $(45.3 \mu \mathrm{g} / \mathrm{g}$ dry root weight from the plant Pentadiplandra brazzeana), showed an $\mathrm{IC}_{50}$ of $92 \mathrm{nM}$ via fluorescent assay and a $\mathrm{Ki}$ of $54 \mathrm{nM}$ via radioactivitybased assay on human sEH (Kitamura et al., 2015). MMU is about 8 -fold more potent than previously reported natural product $\mathrm{sEH}$ inhibitor honokiol (Lee et al., 2014; Kitamura et al., 2015; Figure 2). These findings may explain partly the pharmacological mechanisms of the traditional medicinal use of the root of $P$. brazzeana. Therefore, it is of interest to study whether the use of the root of $P$. brazzeana has beneficial effects in patients with psychiatric and neurological disorders.

Another topic is the systemic anti-inflammatory effects of sEH inhibition or genetic disruption (Liu et al., 2012; Shahabi et al., 2014). Therefore, it is possible that systemic sEH inhibition may play a role in the beneficial effects in CNS disorders through systemic anti-inflammatory actions of $\mathrm{sEH}$ inhibition although

\section{REFERENCES}

Arnold, C., Konkel, A., Fischer, R., and Schunck, W. H. (2010). Cytochrome P450dependent metabolism of omega- 6 and omega- 3 long-chain polyunsaturated fatty acids. Pharmacol. Rep. 62, 536-547. doi: 10.1016/S1734-1140(10)70311-X

Ascherio, P. A., and Schwarzschild, M. A. (2016). The epidemiology of Parkinson's disease: risk factors and prevention. Lancet Neurol. 15, 1257-1272. doi: 10.1016/ S1474-4422(16)30230-7

Bai, Z. G., Bo, A., Wu, S. J., Gai, Q. Y., and Chi, I. (2018). Omega-3 polyunsaturated fatty acids and reduction of depressive symptoms in older adults: a systematic review and meta-analysis. J. Affect. Disord. 241, 241-248. doi: 10.1016/j.jad. 2018.07.057

Bazinet, R. P., and Layé, S. (2014). Polyunsaturated fatty acids and their metabolites in brain function and disease. Nat. Rev. Neurosci. 15, 771-785. doi: 10.1038/ nrn3820

Bloch, M. H., and Qawasmi, A. (2011). Omega-3 fatty acid supplementation for the treatment of children with attention-deficit/hyperactivity disorder symptomatology: systematic review and meta-analysis. J. Am. Acad. Child Adolesc. Psychiatry 50, 991-1000. doi: 10.1016/j.jaac.2011. 06.008

Borlongan, C. V. (2018). Fatty acid chemical mediator provides insights into the pathology and treatment of Parkinson's disease. Proc. Natl. Acad. Sci. U.S.A. 115, 6322-6324. doi: 10.1073/pnas.1807276115

Chang, J. P., Su, K. P., Mondelli, V., and Pariante, C. M. (2018). Omega3 polyunsaturated fatty acids in youths with attention deficit hyperactivity disorder: a systematic review and meta-analysis of clinical trials and biological studies. Neuropsychopharmacology 43, 534-545. doi: 10.1038/npp. 2017.160 further study on the role of systemic anti-inflammation effects of sEH inhibition is needed. It is also suggested that a paracrine role of EET signaling is responsible for a lot of the beneficial effects of EETs (Spector, 2009; Imig, 2016). Therefore, it is possible that up-regulation of $s E H$, which results in decreased paracrine EET signaling that exasperates the disease state although further study on the role of paracrine role of EETs and $\mathrm{sEH}$ is needed.

In conclusion, considering the role of $\mathrm{sEH}$ in the metabolism of EpFAs (e.g., EETs, EDPs), treatment of $\omega-3$ PUFAs in combination with a sEH inhibitor could represent a novel therapeutic approach for psychiatric and neurological disorders. This approach may well bridge the currently unmet medical needs for these CNS disorders.

\section{AUTHOR CONTRIBUTIONS}

The author confirms being the sole contributor of this work and has approved it for publication.

\section{FUNDING}

This study was partly supported by grants from AMED, Japan (to KH, JP18dm0107119).

\section{ACKNOWLEDGMENTS}

I would like to thank my collaborators who are listed as the co-authors of our papers in the reference list.

Chen, C. T., and Bazinet, R. P. (2015). $\beta$-oxidation and rapid metabolism, but not uptake regulate brain eicosapentaenoic acid levels. Prostaglandins Leukot. Essent. Fatty Acids 92, 33-40. doi: 10.1016/j.plefa.2014. 05.007

Cummings, J. L. (1992). Depression and Parkinson's disease: a review. Am. J. Psychiatry 149, 443-454. doi: 10.1176/ajp.149.4.443

Dantzer, R., O'Connor, J. C., Freund, G. G., Johnson, R. W., and Kelley, K. W. (2008). From inflammation to sickness and depression: when the immune system subjugates the brain. Nat. Rev. Neurosci. 9, 46-57. doi: 10.1038/nrn2297

Dehay, B., Bourdenx, M., Gorry, P., Przedborski, S., Vila, M., Hunot, S., et al. (2015). Targeting $\alpha$-synuclein for treatment of Parkinson's disease: mechanistic and therapeutic considerations. Lancet Neurol. 14, 855-866. doi: 10.1016/ S1474-4422(15)00006-X

Denis, I., Potier, B., Heberden, C., and Vancassel, S. (2015). Omega-3 polyunsaturated fatty acids and brain aging. Curr. Opin. Clin. Nutr. Metab. Care 18, 139-146. doi: 10.1097/MCO.0000000000000141

Dowlati, Y., Herrmann, N., Swardfager, W., Liu, H., Sham, L., Reim, E. K., et al. (2010). A meta-analysis of cytokines in major depression. Biol. Psychiatry 67, 446-457. doi: 10.1016/j.biopsych.2009.09.033

Dyall, S. C. (2015). Long-chain omega-3 fatty acids and the brain: a review of the independent and shared effects of EPA, DPA and DHA. Front. Aging Neurosci. 7:52. doi: 10.3389/fnagi.2015.00052

Eyre, H. A., Air, T., Pradhan, A., Johnston, J., Lavretsky, H., Stuart, M. J., et al. (2016). A meta-analysis of chemokines in major depression. Prog. Neuropsychopharmacol. Biol. Psychiatry 68, 1-8. doi: 10.1016/j.pnpbp.2016.02. 006

Farin, F. M., Janssen, P., Quigley, S., Abbott, D., Hassett, C., Smith-Weller, T., et al. (2001). Genetic polymorphisms of microsomal and soluble epoxide hydrolase 
and the risk of Parkinson's disease. Pharmacogenetics 11, 703-708. doi: 10.1097/ 00008571-200111000-00009

Fecchio, C., Palazzi, L., and de Laureto, P. P. (2018). $\alpha$-Synuclein and polyunsaturated fatty acids: molecular basis of the interaction and implication in neurodegeneration. Molecules 23:E1531. doi: 10.3390/molecules23071531

Forte, A., Baldessarini, R. J., Tondo, L., Vázquez, G. H., Pompili, M., and Girardi, P. (2015). Long-term morbidity in bipolar-I, bipolar-II, and unipolar major depressive disorders. J. Affect. Disord. 178, 71-78. doi: 10.1016/j.jad.2015.02.011

Gill, S. S., and Hammock, B. D. (1980). Distribution and properties of a mammalian soluble epoxide hydrase. Biochem. Pharmacol. 29, 389-395. doi: 10.1016/00062952(80)90518-3

Golden, S. A., Covington, H. E., Berton, O., and Russo, S. J. (2011). A standardized protocol for repeated social defeat stress in mice. Nat. Protoc. 6, 1183-1191. doi: 10.1038/nprot.2011.361

Goodarzi, Z., Mrklas, K. J., Roberts, D. J., Jette, N., Pringsheim, T., and Holroyd-Leduc, J. (2016). Detecting depression in Parkinson disease: a systematic review and meta-analysis. Neurology 87, 426-437. doi: 10.1212/ WNL.0000000000002898

Grosso, G., Micek, A., Marventano, S., Castellano, S., Mistretta, A., Pajak, A., et al. (2016). Dietary n-3 PUFA, fish consumption and depression: a systematic review and meta-analysis of observational studies. J. Affect. Disord. 205, 269281. doi: 10.1016/j.jad.2016.08.011

Gumusoglu, S. B., and Stevens, H. E. (2018). Maternal inflammation and neurodevelopmental programming: a review of preclinical outcomes and implications for translational psychiatry. Biol. Psychiatry 85, 107-121. doi: 10. 1016/j.biopsych.2018.08.008

Haapakoski, R., Mathieu, J., Ebmeier, K. P., Alenius, H., and Kivimäki, M. (2015). Cumulative meta-analysis of interleukins 6 and $1 \beta$, tumour necrosis factor $\alpha$ and C-reactive protein in patients with major depressive disorder. Brain Behav. Immun. 49, 206-215. doi: 10.1016/j.bbi.2015.06.001

Hallahan, B., Ryan, T., Hibbeln, J. R., Murray, I. T., Glynn, S., Ramsden, C. E., et al. (2016). Efficacy of omega-3 highly unsaturated fatty acids in the treatment of depression. Br. J. Psychiatry 209, 192-201. doi: 10.1192/bjp.bp.114.160242

Hammock, B. D., Gill, S. S., Stamoudis, V., and Gilbert, L. I. (1976). Soluble mammalian epoxide hydratase: action on juvenile hormone and other terpenoid epoxides. Comp. Biochem. Physiol. B 53, 263-265. doi: 10.1016/03050491(76)90045-6

Harris, T. R., and Hammock, B. D. (2013). Soluble epoxide hydrolase: gene structure, expression and deletion. Gene 526, 61-74. doi: 10.1016/j.gene.2013. 05.008

Hashimoto, K. (2015). Inflammatory biomarkers as differential predictors of antidepressant response. Int. J. Mol. Sci. 16, 7796-7801. doi: 10.3390/ ijms 16047796

Hashimoto, K. (2016). Soluble epoxide hydrolase: a new therapeutic target for depression. Expert Opin. Ther. Targets 20, 1149-1151. doi: 10.1080/14728222. 2016.1226284

Hashimoto, K. (2018). Essential role of Keap1-Nrf2 signaling in mood disorders: overview and future perspective. Front. Pharmacol. 9:1182. doi: 10.3389/fphar. 2018.01182

Hennebelle, M., Otoki, Y., Yang, J., Hammock, B. D., Levitt, A. J., Taha, A. Y., et al. (2017). Altered soluble epoxide hydrolase-derived oxylipins in patients with seasonal major depression: an exploratory study. Psychiatry Res. 252, 94-101. doi: 10.1016/j.psychres.2017.02.056

Hsu, M. C., Tung, C. Y., and Chen, H. E. (2018). Omega-3 polyunsaturated fatty acid supplementation in prevention and treatment of maternal depression: putative mechanism and recommendation. J. Affect. Disord. 238, 47-61. doi: 10.1016/j.jad.2018.05.018

Huang, H. J., Wang, Y. T., Lin, H. C., Lee, Y. H., and Lin, A. M. Y. (2018). Soluble epoxide hydrolase inhibition attenuates MPTP-induced in the nigrostriatal dopaminergic system: involvement of $\alpha$-synuclein aggregation and ER stress. Mol. Neurobiol. 55, 138-144. doi: 10.1007/s12035-017-0726-9

Imaizumi, Y., Okada, Y., Akamatsu, W., Koike, M., Kuzumaki, N., Hayakawa, H., et al. (2012). Mitochondrial dysfunction associated with increased oxidative stress and $\alpha$-synuclein accumulation in PARK2 iPSC-derived neurons and postmortem brain tissue. Mol. Brain 5:35. doi: 10.1186/1756-660 6-5-35

Imig, J. D. (2012). Epoxides and soluble epoxide hydrolase in cardiovascular physiology. Physiol. Rev. 92, 101-130. doi: 10.1152/physrev.00021.2011
Imig, J. D. (2016). Epoxyeicosatrienoic acids and 20-hydroxyeicosatetraenoic acid on endothelial and vascular function. Adv. Pharmacol. 77, 105-141. doi: 10. 1016/bs.apha.2016.04.003

Imig, J. D. (2018). Prospective for cytochrome P450 epoxygenase cardiovascular and renal therapeutics. Pharmacol. Ther. 192, 1-19. doi: 10.1016/j.pharmthera. 2018.06.015

Imig, J. D., and Hammock, B. D. (2009). Soluble epoxide hydrolase as a therapeutic target for cardiovascular diseases. Nat. Rev. Drug Discov. 8, 794-805. doi: 10. $1038 / \mathrm{nrd} 2875$

Jackson-Lewis, V., and Przedborski, S. (2007). Protocol for the MPTP mouse model of Parkinson's disease. Nat. Proc. 2, 141-151. doi: 10.1038/nprot.2006.342

Jamieson, K. L., Endo, T., Darwesh, A. M., Samokhvalov, V., and Seubert, J. M. (2017). Cytochrome P450-derived eicosanoids and heart function. Pharmacol. Ther. 179, 47-83. doi: 10.1016/j.pharmthera.2017.05.005

Jump, D. B. (2002). The biochemistry of n-3 polyunsaturated fatty acids. J. Biol. Chem. 277, 8755-8758. doi: 10.1074/jbc.R100062200

Kalia, L. V., and Lang, A. E. (2015). Parkinson's disease. Lancet 386, 896-912. doi: 10.1016/S0140-6736(14)61393-3

Kamel, F., Goldman, S. M., Umbach, D. M., Chen, H., Richardson, G., Barber, M. R., et al. (2014). Dietary fat intake, pesticide use, and Parkinson's disease. Parkinsonism Relat. Disord. 20, 82-87. doi: 10.1016/j.parkreldis.2013. 09.023

Kitamura, S., Morisseau, C., Inceoglu, B., Kamita, S. G., De Nicola, G. R., Nyegue, M., et al. (2015). Potent natural soluble epoxide hydrolase inhibitors from Pentadiplandra brazzeana baillon: synthesis, quantification, and measurement of biological activities in vitro and in vivo. PLoS One 10:e117438. doi: 10.1371/journal.pone.0117438

Köhler, C. A., Freitas, T. H., Stubbs, B., Maes, M., Solmi, M., Veronese, N., et al. (2018). Peripheral alterations in cytokine and chemokine levels after antidepressant drug treatment for major depressive disorder: systematic review and meta-analysis. Mol. Neurobiol. 55, 4195-4206. doi: 10.1007/s12035-0170632-1

LaChance, L., McKenzie, K., Taylor, V. H., and Vigod, S. N. (2016). Omega-6 to omega-3 fatty acid ratio in patients with ADHD: a meta-analysis. J. Can. Acad. Child Adolesc. Psychiatry 25, 87-96.

Layé, S., Nadjar, A., Joffre, C., and Bazinet, R. P. (2018). Anti-inflammatory effects of omega-3 fatty acids in the brain: physiological mechanisms and relevance to pharmacology. Pharmacol. Rev. 70, 12-38. doi: 10.1124/pr.117.014092

Lee, G. H., Ch, S. J., Lee, S. Y., Lee, J. Y., Ma, J. Y., Kim, Y. H., et al. (2014). Discovery of soluble epoxide hydrolase inhibitors from natural products. Food Chem. Toxicol. 64, 225-230. doi: 10.1016/j.fct.2013.11.042

Lin, P. Y., Chang, C. H., Chong, M. F., Chen, H., and Su, K. P. (2017). Polyunsaturated fatty acids in perinatal depression: a systematic review and meta-analysis. Biol. Psychiatry 82, 560-569. doi: 10.1016/j.biopsych.2017.02. 1182

Lin, P. Y., Huang, S. Y., and Su, K. P. (2010). A meta-analytic review of polyunsaturated fatty acid compositions in patients with depression. Biol. Psychiatry 68, 140-147. doi: 10.1016/j.biopsych.2010.03.018

Liu, Y., Dang, H., Li, D., Pang, W., Hammock, B. D., and Zhu, Y. (2012). Inhibition of soluble epoxide hydrolase attenuates high-fat-diet-induced hepatic steatosis by reduced systemic inflammatory status in mice. PLoS One 7:e39165. doi: 10.1371/journal.pone.0039165

López-Vicario, C., Alcaraz-Quiles, J., García-Alonso, V., Rius, B., Hwang, S. H., Titos, E., et al. (2015). Inhibition of soluble epoxide hydrolase modulates inflammation and autophagy in obese adipose tissue and liver: role for omega3 epoxides. Proc. Natl. Acad. Sci. U.S.A. 112, 536-541. doi: 10.1073/pnas. 1422590112

Luchtman, D. W., Meng, Q., and Song, C. (2012). Ethyl-eicosapentaenoate (E-EPA) attenuates motor impairments and inflammation in the MPTP-probenecid mouse model of Parkinson's disease. Behav. Brain Res. 226, 386-396. doi: 10. 1016/j.bbr.2011.09.033

Ma, M., Ren, Q., Yang, C., Zhang, J. C., Yao, W., Dong, C., et al. (2017). Antidepressant effects of combination of brexpiprazole and fluoxetine on depression-like behavior and dendritic changes in mice after inflammation. Psychopharmacology 234, 525-533. doi: 10.1007/s00213-016-4483-7

Mechawar, N., and Savitz, J. (2016). Neuropathology of mood disorders: do we see the stigmata of inflammation? Transl. Psychiatry 6:e946. doi: 10.1038/tp.2016. 212 
Mello, A. H., Gassenferth, A., Souza, L. R., Fortunato, J. J., and Rezin, G. T. (2014). $\omega-3$ and major depression: a review. Acta Neuropsychiatr. 26, 178-185. doi: $10.1017 /$ neu.2013.52

Miller, A. H., Haroon, E., and Felger, J. C. (2017). Therapeutic implications of brain-immune interactions: treatment in translation. Neuropsychopharmacology 42, 334-359. doi: 10.1038/npp.2016.167

Miller, A. H., Maletic, V., and Raison, C. L. (2009). Inflammation and its discontents: the role of cytokines in the pathophysiology of major depression. Biol. Psychiatry 65, 732-741. doi: 10.1016/j.biopsych.2008.11.029

Miller, A. H., and Raison, C. L. (2016). The role of inflammation in depression: from evolutionary imperative to modern treatment target. Nat. Rev. Immunol. 16, 22-34. doi: 10.1038/nri.2015.5

Mocking, R. J., Harmsen, I., Assies, J., Koeter, M. W., Ruhé, H. G., and Schene, A. H. (2016). Meta-analysis and meta-regression of omega-3 polyunsaturated fatty acid supplementation for major depressive disorder. Transl. Psychiatry 6:e756. doi: $10.1038 /$ tp.2016.29

Morisseau, C., and Hammock, B. D. (2013). Impact of soluble epoxide hydrolase and epoxyeicosanoids on human health. Annu. Rev. Pharmacol. Toxicol. 53, 37-58. doi: 10.1146/annurev-pharmtox-011112-140244

Nestler, E. J., and Hyman, S. E. (2010). Animal models of neuropsychiatric disorders. Nat. Rev. Neurosci. 13, 1161-1169. doi: 10.1038/nn.2647

Newman, J. W., Morisseau, C., and Hammock, B. D. (2005). Epoxide hydrolases: their roles and interactions with lipid metabolism. Prog. Lipid Res. 44, 1-51. doi: 10.1016/j.plipres.2004.10.001

Newman, J. W., Morisseau, C., Harris, T. R., and Hammock, B. D. (2003). The soluble epoxide hydrolase encoded by EPXH2 is a bifunctional enzyme with novel lipid phosphate phosphatase activity. Proc. Natl. Acad. Sci. U.S.A. 100, 1558-1563. doi: 10.1073/pnas.0437724100

Ota, K., and Hammock, B. D. (1980). Cytosolic and microsomal epoxide hydrolases: differential properties in mammalian liver. Science 207, 1479-1481. doi: 10.1126/science.7361100

Polokowski, A. R., Shakil, H., Carmichael, C. L., and Reigada, L. C. (2018). Omega3 fatty acids and anxiety: a systematic review of the possible mechanisms at play. Nutr. Neurosci. 28, 1-11. doi: 10.1080/1028415X.2018.1525092

Qin, X., Wu, Q., Lin, L., Sun, A., Liu, S., Li, X., et al. (2015). Soluble epoxide hydrolase deficiency or inhibition attenuates MPTP-induced Parkinsonism. Mol. Neurobiol. 52, 187-195. doi: 10.1007/s12035-014-8833-3

Raison, C. L., Lowry, C. A., and Rook, G. A. (2010). Inflammation, sanitation, and consternation: loss of contact with coevolved, tolerogenic microorganisms and the pathophysiology and treatment of major depression. Arch. Gen. Psychiatry 67, 1211-1224. doi: 10.1001/archgenpsychiatry.2010.161

Ren, Q., Ma, M., Ishima, T., Morisseau, C., Yang, J., Wagner, K. M., et al. (2016). Gene deficiency and pharmacological inhibition of soluble epoxide hydrolase confers resilience to repeated social defeat stress. Proc. Natl. Acad. Sci. U.S.A. 113, E1944-E1952. doi: 10.1073/pnas.1601532113

Ren, Q., Ma, M., Yang, J., Nonaka, R., Yamaguchi, A., Ishikawa, K. I., et al. (2018). Soluble epoxide hydrolase plays a key role in the pathogenesis of Parkinson's disease. Proc. Natl. Acad. Sci. U.S.A. 115, E5815-E5823. doi: 10.1073/pnas. 1802179115

Sarris, J., Murphy, J., Mischoulon, D., Papakostas, G. I., Fava, M., Berk, M., et al. (2016). Adjunctive nutraceuticals for depression: a systematic review and metaanalyses. Am. J. Psychiatry 173, 575-587. doi: 10.1176/appi.ajp.2016.15091228

Schapira, A. H. V., Chaudhuri, K. R., and Jenner, P. (2017). Non-motor features of Parkinson disease. Nat. Rev. Neurosci. 18, 435-450. doi: 10.1038/nrn.2017.62

Scott-Van Zeeland, A. A., Bloss, C. S., Tewhey, R., Bansal, V., Torkamani, A., Libiger, O., et al. (2014). Evidence for the role of EPHX2 gene variants in anorexia nervosa. Mol. Psychiatry 19, 724-732. doi: 10.1038/mp.2013.91

Sedelis, M., Schwarting, R. K. W., and Huston, J. P. (2001). Behavioral phenotyping of the MPTP mouse model of Parkinson's disease. Behav. Brain Res. 125, 109-125. doi: 10.1016/S0166-4328(01)00309-6

Seidl, S. E., Santiago, J. A., Bilyk, H., and Potashkin, J. A. (2014). The emerging role of nutrition in Parkinson's disease. Front. Aging Neurosci. 6:36. doi: 10.3389/ fnagi.2014.00036

Shahabi, P., Siest, G., and Visvikis-siest, S. (2014). Influence of inflammation on cardiovascular protective effects of cytochrome P450 epoxygenase-derived epoxyeicosatrienoic acids. Drug Metab. Rev. 46, 33-56. doi: 10.3109/03602532. 2013.837916
Shih, P. B. (2017). Integrating multi-omics biomarkers and postprandial metabolism to develop personalized treatment for anorexia nervosa. Prostaglandins Other Lipid Mediat. 132, 69-76. doi: 10.1016/j.prostaglandins. 2017.02.002

Shih, P. B., Yang, J., Morisseau, C., German, J. B., Zeeland, A. A., Armando, A. M., et al. (2016). Dysregulation of soluble epoxide hydrolase and lipidomic profiles in anorexia nervosa. Mol. Psychiatry 21, 537-546. doi: 10.1038/mp.2015.26

Sim, K., Lau, W. K., Sim, J., Sum, M. Y., and Baldessarini, R. J. (2015). Prevention of relapse and recurrence in adults with major depressive disorder: systematic review and meta-analyses of controlled trials. Int. J. Neuropsychopharmacol. 18:pyv076.

Spector, A. A. (2009). Arachidonic acid cytochrome P450 epoxygenase pathway. J. Lipid Res. 50(Suppl.), S52-S56. doi: 10.1194/jlr.R800038-JLR200

Spillantini, M. G., Schmidt, M. L., Lee, V. M. Y., Trojanowsky, J. Q., Jakes, R., and Goedert, M. (1997). $\alpha$-synuclein in Lewy bodies. Nature 388, 839-840. doi: $10.1038 / 42166$

Stoker, T. B., Torsney, K. M., and Barker, R. A. (2018). Emerging treatment approaches for Parkinson's disease. Front. Neurosci. 12:693. doi: 10.3389/fnins. 2018.00693

Sublette, M. E., Ellis, S. P., Geant, A. L., and Mann, J. J. (2011). Meta-analysis of the effects of eicosapentaenoic acid (EPA) in clinical trials in depression. J. Clin. Psychiatry 72, 1577-1584. doi: 10.4088/JCP.10m06634

Swardfager, W., Hennebelle, M., Yu, D., Hammock, B. D., Levitt, A. J., Hashimoto, K., et al. (2018). Metabolic/inflammatory/vascular comorbidity in psychiatric disorders; soluble epoxide hydrolase ( $\mathrm{sEH}$ ) as a possible new target. Neurosci. Biobehav. Rev. 87, 56-66. doi: 10.1016/j.neubiorev.2018. 01.010

Takahashi, S., Mizukami, K., Yasuno, F., and Asada, T. (2009). Depression associated with dementia with Lewy bodies (DLB) and the effect of somatotherapy. Psychogeriatrics 9, 56-61. doi: 10.1111/j.1479-8301.2009. 00292.x

Urquhart, P., Nicolaou, A., and Woodward, D. F. (2015). Endocannabinoids and their oxygenation by cyclo-oxygenases, lipoxygenases and other oxygenases. Biochim. Biophys. Acta 1851, 366-376. doi: 10.1016/j.bbalip.2014. 12.015

Wagner, K., Vito, S., Inceoglu, B., and Hammock, B. D. (2014). The role of long chain fatty acids and their epoxide metabolites in nociceptive signaling. Prostaglandins Other Lipid Mediat. 11, 2-12. doi: 10.1016/j.prostaglandins.2014. 09.001

Wagner, K. M., McReynolds, C. B., Schmidt, W. K., and Hammock, B. D. (2017). Soluble epoxide hydrolase as a therapeutic target for pain, inflammatory and neurodegenerative diseases. Pharmacol. Ther. 180, 62-76. doi: 10.1016/j. pharmthera.2017.06.006

Westphal, C., Konkel, A., and Schunck, W. H. (2015). Cytochrome p450 enzymes in the bioactivation of polyunsaturated Fatty acids and their role in cardiovascular disease. Adv. Exp. Med. Biol. 851, 151-187. doi: 10.1007/978-3319-16009-2_6

World Health Organization [WHO] (2017). Depression. Available at: http://www. who.int/en/news-room/fact-sheets/detail/depression

Wu, Q., Cai, H., Song, J., and Chang, Q. (2017). The effects of sEH inhibitor on depression-like behavior and neurogenesis in male mice. J. Neurosci. Res. 95, 2483-2492. doi: 10.1002/jnr.24080

Yang, C., Qu, Y., Abe, M., Nozawa, D., Chaki, S., and Hashimoto, K. (2017). $(R)$-Ketamine shows greater potency and longer lasting antidepressant effects than its metabolite $(2 R, 6 R)$-hydroxynorketamine. Biol. Psychiatry 82, e43-e44. doi: 10.1016/j.biopsych.2016.12.020

Yang, C., Ren, Q., Qu, Y., Zhang, J. C., Ma, M., Dong, C., et al. (2018). Mechanistic target of rapamycin-independent antidepressant effects of $(R)$-ketamine in a social defeat stress model. Biol. Psychiatry 83, 18-28. doi: 10.1016/j.biopsych. 2017.05.016

Yang, C., Shirayama, Y., Zhang, J. C., Ren, Q., Yao, W., Ma, M., et al. (2015). R-ketamine: a rapid-onset and sustained antidepressant without psychotomimetic side effects. Transl. Psychiatry 5:e632. doi: 10.1038/tp. 2015.136

Young, J. J., Bruno, D., and Pomara, N. (2014). A review of the relationship between pro-inflammatory cytokines and major depressive disorder. J. Affect. Disord. 169, 15-20. doi: 10.1016/j.jad.2014.07.032 
Zhang, J. C., Wu, J., Fujita, Y., Yao, W., Ren, Q., Yang, C., et al. (2014). Antidepressant effects of TrkB ligands on depression-like behavior and dendritic changes in mice after inflammation. Int. J. Neuropsychopharmacol. 18:pyu077. doi: 10.1093/ijnp/pyu077

Zhang, J. C., Yao, W., Dong, C., Yang, C., Ren, Q., Ma, M., et al. (2017a). Blockade of interleukin-6 receptor in the periphery promotes rapid and sustained antidepressant actions: a possible role of gut-microbiota-brain axis. Transl. Psychiatry 7:e1138. doi: 10.1038/tp.2017.112

Zhang, J. C., Yao, W., Dong, C., Yang, C., Ren, Q., Ma, M., et al. (2017b). Prophylactic effects of sulforaphane on depression-like behavior and dendritic changes in mice after inflammation. J. Nutr. Biochem. 39, 134-144. doi: 10.1016/ j.jnutbio.2016.10.004

Zhang, J. C., Yao, W., and Hashimoto, K. (2016a). Brain-derived neurotrophic factor (BDNF)-TrkB signaling in inflammation-related depression and potential therapeutic targets. Curr. Neuropharmacol. 14, 721-731.
Zhang, J. C., Yao, W., Ren, Q., Yang, C., Dong, C., Ma, M., et al. (2016b). Depression-like phenotype by deletion of $\alpha 7$ nicotinic acetylcholine receptor: role of BDNF-TrkB in nucleus accumbens. Sci. Rep. 6:36705. doi: 10.1038/ srep36705

Conflict of Interest Statement: The author declares that the research was conducted in the absence of any commercial or financial relationships that could be construed as a potential conflict of interest.

Copyright (C) 2019 Hashimoto. This is an open-access article distributed under the terms of the Creative Commons Attribution License (CC BY). The use, distribution or reproduction in other forums is permitted, provided the original author(s) and the copyright owner(s) are credited and that the original publication in this journal is cited, in accordance with accepted academic practice. No use, distribution or reproduction is permitted which does not comply with these terms. 